\title{
Genetic and environmental risk factors for sexual distress and its association with female sexual dysfunction
}

\author{
A. Burri ${ }^{1,2 *}$, Q. Rahman ${ }^{2}$ and T. Spector ${ }^{1}$ \\ ${ }^{1}$ Department of Twin Research and Genetic Epidemiology, King's College London, UK \\ ${ }^{2}$ Biological and Experimental Psychology Group, School of Biological and Chemical Sciences, Queen Mary University of London, UK
}

Background. The DSM-V Working Group is currently re-evaluating distress as a primary diagnostic criterion for female sexual dysfunction (FSD). Here, for the first time, we explored the epidemiology of sexual distress and its putative aetiological relationship to FSD by estimating the influence of genetic and environmental risk factors.

Method. Questionnaire data on a representative sample of 930 British female twins using validated scales of FSD and sexual distress were subject to variance components analyses to quantify latent genetic and environmental factors influencing phenotypic variation and covariation. Multiple regression analyses were used to identify other potential risk factors of sexual distress.

Results. Of 319 women with any sexual problems, only 36.5\% reported distress. Of women classified as functional, $16.5 \%$ felt sexual distress. Sexual distress had a heritability of $44 \%$ [ $95 \%$ confidence interval (CI) $0.33-0.54$ ]. Bivariate analysis suggested that the majority (91\% CI 86-99\%) of the covariance between sexual distress and FSD was due to unique environmental effects common to both traits. Associations were found between sexual distress and other risk variables, including relationship dissatisfaction [odds ratio (OR) 1.6, $p<0.001$ ], anxiety sensitivity and obsessivecompulsive symptomatology (OR 1.2, $p<0.01$, for both).

Conclusions. There seems to be a weak phenotypic and genetic basis for including sexual distress as a diagnostic indicator of FSD. Instead, the data indicate that unrelated psychological factors play an important role in sexual distress and tentatively suggest that sexual distress is less a consequence of FSD and more related to general anxiety among women.

Received 1 December 2010; Revised 1 March 2011; Accepted 5 March 2011

Key words: Female sexual dysfunction, genetics, risk factors, sexual distress, twins.

\section{Introduction}

Female sexual dysfunction (FSD) is an umbrella term, comprising sexual desire, arousal, orgasm and pain disorders. Several inconsistent classification systems for FSD have been proposed, all of which are heavily based on the original conceptualization of the human sexual response (Masters \& Johnson, 1966). Because of the inconsistencies of these classification systems, a consensus-based definition and classification system for FSD, offering guidelines for clinical evaluation and treatment, was designed by the International Consensus Development Conference (Basson et al. 2000, 2004). Accordingly, a woman should show evidence of significant personal distress, characterized

* Address for correspondence: Dr A. Burri, Biological and Experimental Psychology Group, School of Biological and Chemical Sciences, Queen Mary University of London, Mile End Road, London E1 4NS, UK.

(Email: a.burri@qmul.ac.uk) by negative feelings and anxiety about one's sexuality or sexual activities, in relation to her sexual problem to qualify for the diagnosis of FSD. In 1994, 'marked distress or interpersonal difficulty' was added to the criteria sets for all the sexual dysfunctions in DSM-IV to delineate the dysfunction from a normal variant of functioning (APA, 1994). However, quantitative evidence questions the utility of personal sexual distress as a primary diagnostic indicator of FSD and experts have been unable to reach consensus on whether or not to include distress in the symptom criteria for the diagnosis of sexual dysfunction (Althof, 2001; Bancroft et al. 2003; Segraves et al. 2007; Witting et al. 2008). The DSM-V Working Group is currently reevaluating distress as a primary diagnostic criterion in view of the evidence that some persons with sexual dysfunction are not distressed by it (Graham, 2010).

Several studies have investigated distress associated with FSD (Bancroft et al. 2003; Oberg et al. 2004; King et al. 2007; Hayes et al. 2008; Witting et al. 2008). 
A consistent finding across these studies has been that sexual problems, independent of their degree of severity, do not always cause distress. Shifren et al. (2008) reported that the prevalence of low arousal decreased from $25.3 \%$ to $3.3-6 \%$ (depending on age) when including distress as assessed with the Female Sexual Distress Scale (FSDS; Derogatis et al. 2002) whereas Dennerstein \& Hayes (2005) observed that $16 \%$ of women aged $20-49$ years had low sexual desire compared with only $7 \%$ when personal distress was included as a diagnostic criterion. King et al. (2007) reported that of $38 \%$ of women deemed to have an ICD-10 diagnosis of a sexual dysfunction, only $18 \%$ of women received a diagnosis and also perceived that they had a problem (see Graham, 2010, for a comprehensive review). Correlational evidence further suggests that the inconsistency in such diagnostic validation studies is in part due to a failure to consider important demographic, psychological and interpersonal 'risk' factors, which are robustly correlated with FSD symptoms in non-clinical and clinical, community and cross-sectional populations (Bancroft et al. 2003; Witting et al. 2008). These factors include general relationship duration and satisfaction, partner compatibility, the presence of one or more sexual disorders in a partner, and general psychological and physical health. A more recent study further found attachment anxiety to be an important moderator of the association between FSD and subjective sexual distress in women (Stephenson \& Meston, 2010).

Despite these findings, the relationship between levels of sexual functioning and sexual distress remains unclear and further work in this area is vital for a re-evaluation of sexual distress as a putative diagnostic criterion for FSD.

To explore why some women report sexual distress concurrently with FSD symptoms whereas others do not, we assessed the prevalence of sexual distress in a representative sample of British female twins with and without FSD-type symptoms. A strong design should include tests for possible familial confounding (genetic and non-genetic) of the association between sexual distress and FSD symptoms, and also test for the relationships between related demographic and psychological risk factors and sexual distress. To do this, we used twin modelling to quantify the contribution of genetic, shared and non-shared environmental factors to the variance of reported sexual distress and FSD, and to any covariance between them. We used regression methods to help to identify previously reported risk factors for FSD and sexual distress, including relationship satisfaction, history of abuse, personality, emotional intelligence, anxiety and the related concept of obsessive-compulsive behaviour.

\section{Method}

Sample

Subjects for this research project were female monozygotic (MZ) and dizygotic (DZ) twins enlisted in the UK Adult Twin Registry (TwinsUK; Spector \& Williams, 2006). TwinsUK was started in 1993 and comprises a cohort of unselected volunteer Caucasian twins. All volunteers in the registry have been recruited through successive national media campaigns in the UK and Ireland and from other twin registers. The twins have undergone extensive clinical investigations and have been shown to be comparable with age-matched singletons in terms of disease prevalence, lifestyle characteristics and also sexual behaviour and functioning (e.g. Andrew et al. 2001; Burri et al. 2009). Data collection was carried out in waves. Collection of responses to the Female Sexual Function Index (FSFI) and the Female Sexual Distress Scale (FSDS) was carried out in 2008/2009 (Rosen et al. 2000; Derogatis et al. 2002). Data collection of the potential risk factors was carried out in waves between 2007 and 2009. The study targeted a subsample of 3154 (29.7\% of twins from the entire TwinsUK registry; ages 25-85 years, mean age 56.2 years) female twin individuals who had previously filled in sexualityrelated questionnaires and stated their willingness to participate in studies of this and a similar nature. The study was approved by the St Thomas' Hospital Research Ethics Committee and all twins provided informed consent. The subjects were not selected on the basis of variables being studied (such as the presence or absence of sexual dysfunctions) and were unaware of the specific research aims.

\section{Measures}

\section{FSD and sexual distress}

Our primary measures included the FSFI to measure FSD symptoms and the FSDS to index sexual distress. The 19-item FSFI is an easy-to-administer self-report questionnaire providing multi-dimensional assessment of female sexual function in the past 4 weeks (Rosen et al. 2000). The six dimensions of the FSFI (desire, arousal, lubrication, orgasm, satisfaction and pain) reflect the different subtypes of FSD, apart from sexual satisfaction, for which no separate diagnostic category of dysfunction has yet been provided. Details on response options, domain score computation and domain factor weights can be found in Rosen et al. (2000). Low scores on the FSFI indicate more problems with sexual function and high scores indicate fewer problems. The questionnaire has received extensive psychometric evaluation in clinical and non-clinical 
samples. In the initial evaluation study on two groups of women, including subjects with female sexual arousal disorder (FSAD, $n=128$ ) and age-matched controls $(n=131)$, the questionnaire showed a high degree of internal consistency (Cronbach's $\alpha \geqslant 0.82$ ) and high test-retest reliability for each domain $(r=0.79-0.86)$ (Rosen et al. 2000). It differentiated well between sexually dysfunctional and non-dysfunctional women on all different dimensions. In addition, good construct validity was demonstrated by highly significant mean difference scores between the FSAD and the control group for each of the domains ( $p<$ 0.001). The questionnaire was further successfully cross-validated in several samples of women with mixed sexual dysfunctions (Meston, 2003). In addition, a diagnostic cut-off score (26.55) for potential classification of total FSD, allowing differentiation of women with and without sexual dysfunction, was developed using means of standard receiver operating characteristics curves (Wiegel et al. 2005).

The FSDS is the standard and common 12-item instrument used to assess subjective distress associated with FSD (Derogatis et al. 2002). Response options are on a five-point scale, ranging from $0=$ never to $4=$ always, with a higher score indicating increased sexual distress. The psychometric properties of the instrument have been evaluated and replicated in several clinical trials. Initial validation of the scale on a sample of 78 women has shown a high degree of testretest reliability $(r=0.91)$, good validity and internal consistency (Cronbach's $\alpha \geqslant 0.8$ ) (Derogatis et al. 2002, 2008). The measure also discriminated well between sexually functional and dysfunctional women and demonstrated a high sensitivity to treatment-induced changes from baseline to treatment termination.

\section{Anxiety and obsessive-compulsive behaviour}

Obsessive-compulsive symptoms (including distress related to having obsessions) was measured using the 42-item Obsessive Compulsive Inventory (OCI; Foa et al. 2002). The questionnaire is divided into seven subscales with response options ranging from $0=$ never to $4=$ almost always. A total score can be calculated by adding the scores for all items. The OCI has shown excellent internal consistency $(r=0.93)$ and high test-retest reliability in an obsessive-compulsive disorder (OCD) sample $(r=0.84-0.87)$ and in nonpatient controls ( $r=0.90-0.89$ ) (Foa et al. 2002).

Anxiety sensitivity was measured with the 16item Anxiety Sensitivity Index (ASI; Reiss et al. 1986). Anxiety sensitivity is defined as the fear of arousalrelated sensations, arising from beliefs that these anxiety-related sensations have harmful consequences. Items are responded to on a five-point
Likert-type scale ranging from $0=$ very little to $4=$ very much. The psychometric properties and predictive validity of the widely used instrument have been well established and several studies have provided replicated evidence that the ASI has adequate internal consistency ( $\alpha=0.81-0.94)$, a good degree of test-retest reliability $(r=0.71-0.75)$ and a high degree of inter-item relatedness (Peterson \& Reiss, 1992; Peterson \& Plehn, 1999).

\section{Personality and emotional intelligence}

The Big Five personality dimensions were assessed using the Ten-Item Personality Index (TIPI; Woods \& Hampson, 2005). The TIPI is a 10-item measure designed to quickly assess the different personality dimensions with optimized validity. Hence, the use of the TIPI is mainly indicated for situations where short measures are needed and personality is not the primary topic of interest. Response options are on a seven-point Likert scale ranging from $1=$ disagree strongly to $7=$ agree strongly. In a US study on 1787 undergraduates, the instruments reached adequate levels in terms of convergence with widely used multiitem Big-Five measures (e.g. the Big Five Inventory, BFI) in self-, observer and peer reports (mean of $r=0.77)$ and good test-retest reliability $(r=0.62-0.77)$ (Woods \& Hampson, 2005).

Emotional intelligence was measured with the Trait Emotional Intelligence Questionnaire-Short Form (TEIQue-SF; Petrides \& Furnham, 2006). The 30 items of the TEIQue-SF are responded to on a seven-point Likert scale ranging from $1=$ completely disagree to $7=$ completely agree. A total emotional intelligence score can be derived by adding the point values for each item together. In a study on 167 subjects, the TEIQue-SF has shown to have high levels of internal consistency (Cronbach's $\alpha>0.80$ ) and good construct validity. Additional validity evidence can be found in Petrides \& Furnham (2006).

\section{Other demographic, psychological and interpersonal 'risk factor' measures}

Demographic variables on all twins including age, current marital status and years of education were obtained from the TwinsUK database. Mental and physical health were both classified as dichotomous traits on the basis of a subject's response to the question: 'Do you suffer from any conditions: (a) medical; (b) psychological?' Events of physical, emotional and sexual abuse were responded to on a no(0)/yes(1) dichotomous scale. Current relationship satisfaction was assessed using a self-constructed questionnaire, with response options ranging from 'very satisfied' (1) to 
'not satisfied at all' (6). Events of physical, emotional and sexual abuse that had been reported previously as potential risk factors for FSD were assessed with a single question with response option no (0)/yes (1).

\section{Statistical analysis}

Data handling and all statistical analyses were carried out using Stata software (StataCorp, 2007). Genetic modelling was carried out with Mx software (Neale et al. 2006). For regression analyses, FSD and sexual distress were treated as dichotomous traits $(0=\mathrm{no} /$ $1=$ yes) according to the previously suggested cut-off scores (26.55 for the FSFI and 15 for the FSDS (Rosen et al. 2000 ; Derogatis et al. 2002) For genetic modelling, continuous, age-regressed measures were used. A $p$ value $<0.05$ or odds ratios (OR) with a $95 \%$ confidence interval (CI) not including ' 1 ' were considered statistically significant, unless stated otherwise. All tests were two-tailed. In all regression analyses, nonindependence of twin pairs was accounted for by using the cluster function for familial relatedness, which is a form of conditional regression. Bonferroni correction was used to account for multiple testing in the multivariate regression analysis.

\section{Sample characteristics and risk factor measures}

Simple logistic regression analyses were conducted to investigate effects of potential 'risk factors' (see Method section) on sexual distress. Significant variables were then entered into multiple regression models as independent variables. However, when constructing this model, a high intercorrelation between 'emotional stability' and 'emotional intelligence' was detected $(r=0.81)$. Among this variable pair, only the one showing stronger unadjusted association with the outcome measure was entered into the models. A stepwise backward approach was used. Unpaired two-tailed $t$ tests were applied to assess mean differences in all continuous variables between $\mathrm{MZ}$ and DZ twins. Dichotomous and categorical data were expressed as percentages and comparisons between the two zygosity groups were conducted using two-sample tests of proportions.

\section{Genetic modelling}

Standard methods of quantitative genetic analysis were used to model latent genetic and environmental factors influencing sibling covariance in sexual distress for $\mathrm{MZ}$ and $\mathrm{DZ}$ twins. The twin design makes use of the fact that MZ twins share identical genotypes $(>99.9 \%$ ) whereas DZ twins share on average $50 \%$ of their segregating genes, and is based on the fundamental assumption that MZ and DZ twin pairs share the intra-pair environment to the same degree (Neale \& Cardon, 1992). Genetic model fitting was used for the decomposition of the observed phenotypic variance $(\mathrm{P})$ into additive $(\mathrm{A})$ and dominant $(\mathrm{D})$ genetic effects, and common $(\mathrm{C})$ and unique environmental (E) effects (Posthuma et al. 2003). Initial assessment of the components (A, D, C and E) may suggest non-significant values in one or more component. Further analysis can determine the significance of each factor as components of the observed variance by removing each sequentially from the full model and testing the deterioration in fit of the various nested models, using the likelihood ratio test. In addition, the Akaike Information Criteria (AIC $=\chi^{2}-2$ df) was considered, with lower values indicating the most suitable model ( $\mathrm{Hu} \&$ Bentler, 1995). The AIC combines the goodness of fit of a model (the discrepancy of expected to observed covariance matrixes) with its simplicity, resulting in a measure of parsimony. The most parsimonious model was then used to estimate heritability.

Genetic modelling was extended to multivariate model fitting to quantitatively explore phenotypic covariation between sexual distress and FSD and to test whether the same genetic and environmental factors contribute to their covariance (Loehlin, 1995; Posthuma et al. 2003). A Cholesky decomposition was considered, which includes two independent genetic and environmental factors. The first factor loads on both traits, the second factor loads only on the other trait. This provides the fullest potential explanation of the data. To determine the degree of shared genetic and environmental influences, the most parsimonious model was selected on the basis of the lowest AIC. Here we present both the estimated genetic covariance between the traits as a proportion of the total phenotypic covariance (bivariate heritability) and the proportion of the total genetic variance for the traits (genetic correlation). Bivariate heritability is interpreted as the proportion of a phenotypic correlation that can be indirectly attributed to additive polygenic causal factors in a population, whereas the genetic correlation reflects the proportion of total genetic variance attributable to shared additive polygenic effects between the traits.

\section{Results}

Sample characteristics and prevalence of sexual distress

Of the 1589 women who filled in the questionnaires, nine who reported never having been sexually active were excluded from further analyses. Women with 
Table 1. Differences in lifestyle and behavioural risk factors and demographic characteristics for women reporting having been sexually active at the time of data assessment and women reporting no sexual activity. Significant differences are shown in italics

\begin{tabular}{|c|c|c|c|c|c|c|c|}
\hline & \multicolumn{3}{|c|}{ Sexually active $(n=930)$} & \multicolumn{4}{|c|}{ Sexually inactive $(n=559)$} \\
\hline & Mean & S.D. & Range & Mean & S.D. & Range & $p$ value $^{\mathrm{a}}$ \\
\hline Age (years) & 52.93 & 10.89 & $18-85$ & 61.67 & 10.72 & $20-82$ & * \\
\hline Education (years) & 10.89 & 2.97 & $6-31$ & 10.66 & 2.72 & $6-32$ & 0.14 \\
\hline Extraversion & 3.36 & 1.57 & $1-7$ & 3.47 & 1.58 & $1-7$ & 0.19 \\
\hline Agreeableness & 2.44 & 1.12 & $1-6$ & 2.43 & 1.14 & $1-6$ & 0.86 \\
\hline Emotional stability & 3.25 & 1.37 & $1-7$ & 3.37 & 1.43 & $1-7$ & 0.11 \\
\hline Conscientiousness & 1.96 & 0.96 & $1-6.5$ & 1.96 & 0.98 & $1-6.5$ & 0.87 \\
\hline Openness & 3.08 & 1.20 & $1-7$ & 3.14 & 1.32 & $1-7$ & 0.36 \\
\hline Emotional intelligence & 156.94 & 23.76 & 59-203 & 154.44 & 24.93 & 59-207 & 0.87 \\
\hline Relationship satisfaction & 5.82 & 0.78 & $1-6$ & 3.33 & 2.14 & $1-6$ & $*$ \\
\hline AS & 13.44 & 9.23 & $0-60$ & 14.78 & 10.61 & $0-59$ & 0.29 \\
\hline \multirow[t]{2}{*}{ OCB } & 6.92 & 9.02 & $0-79$ & 7.10 & 9.92 & $0-88$ & 0.10 \\
\hline & $n$ & $\%$ & & $n$ & $\%$ & & $p$ value ${ }^{\mathrm{b}}$ \\
\hline \multicolumn{8}{|l|}{ Marital status } \\
\hline Single & 40 & 4.31 & & 47 & 8.41 & & $*$ \\
\hline Married & 492 & 52.90 & & 264 & 47.23 & & * \\
\hline In relationship & 344 & 36.99 & & 124 & 22.18 & & * \\
\hline Divorced & 37 & 3.98 & & 84 & 15.02 & & $*$ \\
\hline Widowed & 17 & 1.83 & & 40 & 7.16 & & * \\
\hline Children & 863 & 92.79 & & 504 & 90.16 & & 0.07 \\
\hline Sexual abuse & 91 & 9.78 & & 67 & 11.98 & & 0.18 \\
\hline Physical abuse & 96 & 10.33 & & 75 & 13.42 & & 0.07 \\
\hline Emotional abuse & 201 & 21.06 & & 149 & 26.65 & & 0.08 \\
\hline Medical conditions & 138 & 14.83 & & 91 & 16.27 & & 0.45 \\
\hline Psychological conditions & 85 & 9.14 & & 67 & 11.98 & & 0.07 \\
\hline
\end{tabular}

AS, Anxiety sensitivity; OCB, obsessive-compulsive behaviour ; S.D., standard deviation.

${ }^{\text {a }}$ Unpaired two-tailed $t$ tests were used to test for mean differences.

$\mathrm{b}$ Two-sample tests of proportions were used to explore differences in response frequencies.

${ }^{*} p$ value $<0.005$.

more than five of the 19 items in the FSFI and/or more than two items of the FSDS missing were further dropped from the sample (Witting et al. 2008). A total of 559 women reported not having engaged in sexual activities during the measurement period and therefore had to be excluded from the analyses as well, resulting in a sample of 930 women, comprising $119 \mathrm{MZ}$ pairs, $67 \mathrm{DZ}$ pairs and 558 single twins.

The characteristics of recently sexually active and sexually inactive women are shown in Table 1 . The mean age of participants in the total sample was 56.3 years (s.D. $=11.63$, range 18-85). Substantial differences in sociodemographic variables were found between women who reported sexual activity during the past 4 weeks compared with women who did not (Table 1). Recently sexually inactive women were significantly older (61 v. 52 years, $p<0.001$ ) and more often single
(8.41\% v. $4.31 \%, p<0.005)$, divorced $(15.02 \%$ v. $3.98 \%$, $p<0.001)$ or widowed $(7.16 \% v .1 .83 \%, p<0.001)$ than sexually active women. In terms of potential lifestyle and behavioural risk factors for FSD, recently sexually inactive women were not found to be significantly different from sexually active women, apart from being less satisfied with their relationship $(p<0.001)$.

The MZ and DZ twin groups were well matched for most sociodemographic variables and potential risk factors for sexual distress, except for the marital status 'divorced' (6.5\% v. 9.6\%, $p<0.01)$. No significant differences in prevalence of sexual distress and FSD were found between MZ and DZ twins. A total of $34.31 \%$ of women met the criteria of FSD (based on the FSFI total score cut-off of 26.55). A detailed discussion on prevalence rates of FSD in our study sample can be found elsewhere (Burri \& Spector, in press). 


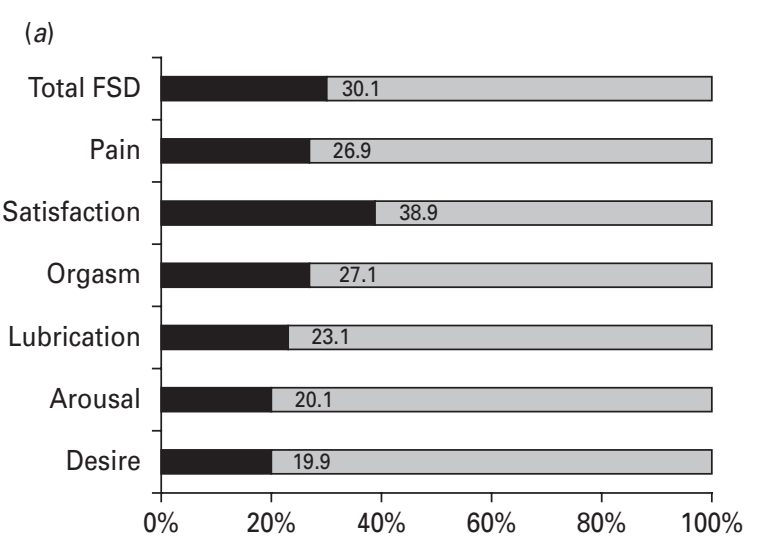

(b)

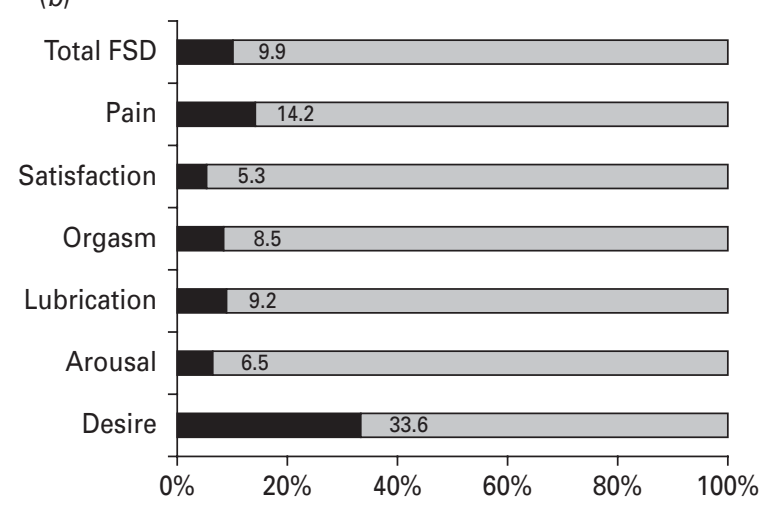

Fig. 1. Percentages of $(a)$ sexually functioning women and (b) sexually 'dysfunctional' women who reported sexual distress as defined by Female Sexual Function Index (FSFI) scores (e.g. of all women reporting sexual pain problems, $26.9 \%$ report sexual distress).

Overall, $26.6 \%$ of the women in our study reported sexual distress (Table 1). All associations between the subdomains of the FSFI and sexual distress were significant $(r=0.11-0.42)$. The strongest association was found for sexual satisfaction and the lowest for hypoactive desire. To meet the criteria of FSD, individuals must feel significant distress over their sexual problems. However, approximately one-third of women scored above the FSDS cut-off, therefore reporting sexual distress (ranging from 25.5\% to $41.9 \%$ depending on the subtype of FSD) (Fig. 1). In addition, a substantial number of women classified as 'functional' according to the FSFI felt markedly distressed about their levels of sexual functioning (ranging from $10.4 \%$ to $19.1 \%$ depending on the subtype of FSD).

\section{Risk factors for sexual distress}

Variables significantly correlated with sexual distress in the univariate regression were entered into a multiple regression model to investigate whether they were independently associated with sexual distress (Table 2). Age was negatively correlated with reported sexual distress $(p<0.001)$. The strongest association was found with reported suffering from a psychological condition (OR 2.1, 95\% CI 1.53-2.98, $p<0.001$ ); women reporting the latter having a twofold significant risk of sexual distress. The second strongest association was with relationship satisfaction; less satisfied women were significantly more likely to report sexual distress (OR 1.56, 95\% CI 1.43-1.72, $p<0.001)$. Anxiety sensitivity and obsessive-compulsive behaviour were also both significantly associated with sexual distress (OR 1.2, 95\% CI 1.05-1.34 and OR 1.2, 95\% CI 1.01-1.4, $p<0.01$ for both), with high scores on these measures associated with higher levels of sexual distress. There were no significant associations between sexual distress, emotional intelligence and the Big Five personality scores.

\section{Genetic modelling}

Variance component modelling revealed that an $\mathrm{AE}$ model, ascribing the total variance in liability to report sexual distress to additive genetic factors and nonshared environmental factors, was the best fitting. This revealed a heritability of $46 \%$ (95\% CI 0.33 0.54) (Table 3). Non-shared environmental factors (including random error) accounted for up to $54 \%$ of the total variation in sexual distress. Similarly, an AE model was the best fitting for overall FSD, resulting in a heritability estimate of $29 \%$ (95\% CI $0.17-0.39)$ (Table 3).

The cross-twin cross-trait correlations deviated notably from phenotypic correlations $\left(r_{\mathrm{p}}=-0.53\right)$, pointing towards non-shared environmental influence on phenotypic correlations, and were approximately twice as large in $\mathrm{MZ}(r=0.15)$ compared with DZ twin pairs $(r=0.07)$, indicating no influence of common environmental factors. ACE estimates from the bivariate model were in line with those from the univariate analyses. The bivariate model suggested that approximately 9\% (95\% CI 4-15) of the covariance between sexual distress and FSD was due to additive genetic factors, with the remaining $91 \%$ (95\% CI 86-99) attributable to non-shared environmental factors (Table 4). Common environmental components for the phenotypic variance and the covariance between sexual distress and FSD were negligible. The genetic correlation for the full model was -0.28 (95\% CI 19-30). The Cholesky model-fitting results suggested one (weak) genetic factor loading on sexual distress (Fig. 2). The non-shared environmental influences were more variable specific but suggested one 
Table 2. Significant results from the univariate and multivariate regression analyses of potential risk factors for sexual distress in our study population. Only variables significantly associated with sexual distress in the univariate analyses are displayed. Significant results of the multivariate model are shown in italics. Non-independence of twin pairs was accounted for using the cluster function for familial relatedness. In the multivariate analyses, all p values were Bonferroni corrected to account for multiple testing

\begin{tabular}{|c|c|c|c|c|c|c|c|c|}
\hline & \multicolumn{4}{|c|}{ Univariate regression } & \multicolumn{4}{|c|}{ Multivariate regression } \\
\hline & OR & $95 \% \mathrm{CI}$ & $p$ & $R^{2}$ & OR & $95 \% \mathrm{CI}$ & $p$ & $R^{2}$ \\
\hline Sexual distress & & & & & & & & 0.17 \\
\hline Age & 0.96 & $0.94-0.98$ & $* *$ & 0.02 & 0.96 & $0.93-0.99$ & $* *$ & \\
\hline Menopause & 0.47 & $0.31-0.71$ & $* *$ & 0.01 & - & - & - & \\
\hline Medical conditions & 1.51 & $1.01-2.27$ & $*$ & 0.01 & 0.85 & $0.31-1.36$ & 0.47 & \\
\hline Psychological conditions & 2.20 & $1.43-3.40$ & $* *$ & 0.11 & 2.14 & $1.53-2.98$ & $* *$ & \\
\hline Emotional stability & 1.21 & $1.06-1.37$ & $* *$ & 0.01 & - & - & - & \\
\hline Emotional intelligence & 0.98 & $0.97-0.99$ & $* *$ & 0.01 & 0.99 & $0.98-1.01$ & 0.11 & \\
\hline Relationship satisfaction & 1.59 & $1.45-1.75$ & $* *$ & 0.13 & 1.56 & $1.43-1.72$ & $* *$ & \\
\hline Anxiety sensitivity & 1.30 & $1.01-1.75$ & $* *$ & 0.02 & 1.20 & $1.05-1.34$ & * & \\
\hline Obsessive-compulsive behaviour & 1.20 & $1.00-1.42$ & ** & 0.01 & 1.20 & $1.01-1.47$ & * & \\
\hline
\end{tabular}

OR, Odds ratio; $\mathrm{CI}$, confidence interval.

$R^{2}$ indicates the phenotypic variance explained by the potential risk factor(s).

${ }^{*} p<0.01,{ }^{* *} p<0.001$.

Table 3. Results of the univariate model fitting of sexual distress and overall female sexual dysfunction (FSD). Comparison of different restricted submodels to the nested model revealed a best-fitting AE model for sexual distress and overall FSD, ascribing $44 \%$ and $28 \%$, respectively of the phenotypic variance to additive genetic effects

\begin{tabular}{lllllll}
\hline & $\mathrm{A}(95 \% \mathrm{CI})$ & $\mathrm{C} / \mathrm{D}(95 \% \mathrm{CI})$ & $\mathrm{E}(95 \% \mathrm{CI})$ & $\chi^{2}(\mathrm{df})$ & $p$ & AIC \\
\hline Sexual distress & $0.44(0.33-0.54)$ & - & $-0.56(0.45-0.66)$ & $7.50(901)$ & 0.01 & 1623.45 \\
FSD & $0.28(0.17-0.39)$ & - & $0.72(0.60-0.82)$ & $0.00(862)$ & 0.94 & 1493.37 \\
\hline
\end{tabular}

A, Additive genetic effects; D, dominant genetic effects; C, common environmental effects; E, unique environmental effects and random error; CI, confidence interval; AIC, Akaike Information Criterion. AIC describes the model with best goodness-offit combined with parsimony. The submodel with the lowest AIC is the best fitting; $\chi^{2}$, goodness-of-fit statistic; df, degrees of freedom; $p$, probability that Dc2 is zero.

weak environmental factor loading on both FSD and sexual distress.

\section{Discussion}

According to the phenotypic level analyses, the present data are consistent with several previous studies showing that women reporting impaired sexual functioning do not always report feeling distressed by it (e.g. Oberg et al. 2004; Witting et al. 2008). The prevalence of sexual distress here $(26.6 \%)$ was comparable to that reported in two US national probability samples (24.4\% and 22.2\%; Bancroft et al. 2003; Shifren et al. 2008). In our study only up to one in three women reported sexual distress in conjunction with sexual problems, whereas one in eight felt sexual distress even without a concomitant sexual problem. The present study also showed, for the first time, that there were significant genetic influences on sexual distress (heritability was $46 \%$ ) and substantial non-shared environmental influences (and no effect of shared environmental influences). FSD was also found to be heritable (at $29 \%$ ), which is consistent with previous twin studies showing a moderate heritability to sexual problems (Dawood et al. 2005; Dunn et al. 2005; Witting et al. 2009). Witting et al.'s (2009) report is of note because they used a population-based sample of 6446 Finnish female twins. They reported additive factors underlying the specific subdomains of FSD in the range $0-15 \%$ and non-additive genetic effects in the range of $0-24 \%$. Our higher heritability estimate for FSD could be due to the greater statistical power in Witting et al.'s study allowing the detection of dominant genetic effects, in addition to their smaller age 
Table 4. Proportion of phenotypic correlations (bivariate heritability) due to additive genetic $(A)$, shared environmental $(C)$ and non-shared environmental (E) influences; and genetic, shared environmental and non-shared environmental correlations

\begin{tabular}{lc}
\hline & FSD - Sexual distress \\
\hline$r_{\mathrm{p}}$ & -0.53 \\
\hline Proportion of $r_{\mathrm{p}}$ due to & \\
$\mathrm{A}$ & 0.09 \\
$\mathrm{C}$ & 0.00 \\
$\mathrm{E}$ & 0.91 \\
Correlations & \\
$r_{\mathrm{A}}$ & -0.28 \\
$r_{\mathrm{C}}$ & - \\
$r_{\mathrm{E}}$ & -0.72 \\
\hline
\end{tabular}

$r_{\mathrm{p}}$, Phenotypic correlation; $r_{\mathrm{A}}, r_{\mathrm{C}}, r_{\mathrm{E}}$, genetic, shared environmental and non-shared environmental correlations respectively.

$N($ monozygotic $)=119$ pairs, $n($ dizygotic $)=67$ pairs .

range (mean age 29.3 years, range 18-49 years). Despite genetic influences on both sexual distress and FSD, only one-tenth of the covariance between these two traits was attributable to additive genetic effects. Instead, the covariance was primarily due to nonshared environmental factors. The high environmental correlation $(72 \%)$ between the two traits further indicates that most of this was attributable to common environmental covariance, suggesting that similar environmental factors, rather than genetic factors, underlie both traits.

Several candidate factors have been reported in the literature that may help explain this putative 'common environmental' aetiology for both sexual distress and FSD, including relationship satisfaction (Witting et al. 2008) and psychopathological symptomatology (Dunn et al. 1999; Frohlich \& Meston, 2002; Abdo et al. 2004). These are also consistent with our findings regarding related 'risk factors' for sexual distress. Women in our study who were less satisfied with their current relationship were approximately 1.5 times more likely to report sexual distress. It is unclear whether this association is mediated by partner compatibility, as demonstrated by Witting et al. (2008), but both our results and theirs suggest that women dissatisfied with their relationships report more sexual distress, independent from their actual level of sexual functioning. We also found a novel association between self-reported anxiety and obsessive-behaviour symptoms and sexual distress. These findings parallel previous studies that reported an association between high anxiety and depression scores and sexual problems in epidemiological and community samples of

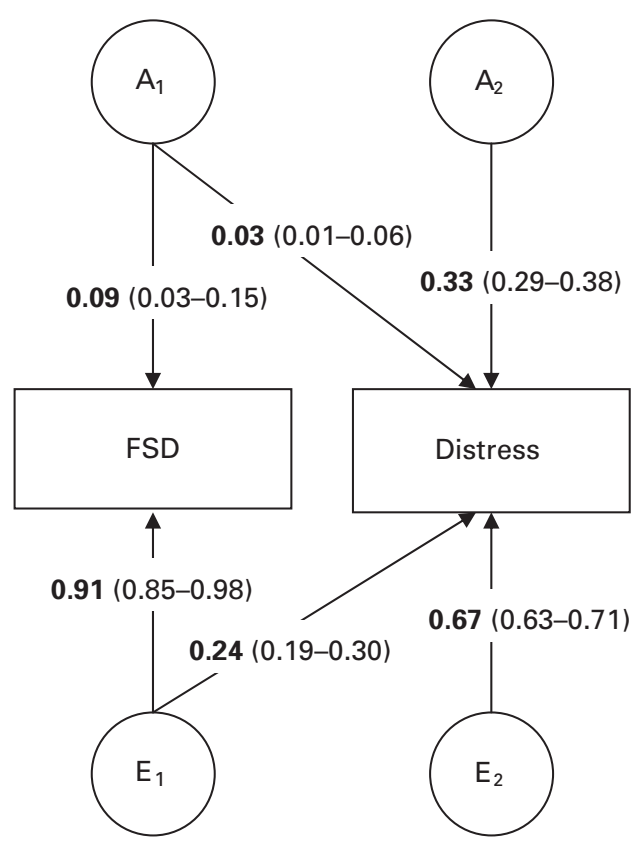

Fig. 2. Illustration of the Cholesky decomposition for the covariance in female sexual dysfunction (FSD) and sexual distress. The variance of 119 monozygotic (MZ) and 67 dizygotic (DZ) twin pairs was decomposed to additive genetic (A1-A2) and unshared environmental (E1-E2) factors. Standardized factor loadings with $95 \%$ confidence interval (CI) for FSD and sexual distress are displayed.

women (Dunn et al. 1999; Frohlich \& Meston, 2002; Hayes et al. 2008). These studies suggest that generalized anxiety or affective problems increase maladaptive attention towards quality of sexual performance and body image that may be imperfectly related to actual sexual activity.

Overall, our data indicate that researchers (both basic and clinical) need to take a more nuanced approach to studying sexual dysfunction and sexual distress. Prevalence rates for FSD in literature are in the region of $60 \%$ (depending on sample characteristics and assessment methods), a very high level of disease prevalence for a variable trait such as the female sexual response. Yet, here we have shown that not every woman suffering from FSD also reports sexual distress, a key criteria in the diagnosis of FSD. On the contrary, some women classified as functional according to the FSFI felt distressed about their levels of sexual functioning. The relevance of sexual distress may depend, in part, on the dysfunction itself in addition to developmental context. For example, low sexual desire may be completely independent of distress in post-menopausal women whereas problems in lubrication may be strongly correlated with distress in younger, more sexually active women. Researchers should consider using sophisticated multivariate 
approaches (e.g. discriminant function techniques) to quantify the differences between different dysfunctions in the context of sexual distress in different populations of women.

The present findings should be considered in light of several methodological limitations. A general study limitation is the fact that we were not able to directly determine the direction of causality between our variables, in particular between FSD and sexual distress. Further prospective research is needed to investigate the link between FSD and sexual distress and to determine whether variance in candidate ' risk' factors can track temporal changes in sexual distress. Our participants were somewhat older than previous work (mean age $=56.2$ years). Using the average age of menopause (50 years) to classify women into pre- and postmenopausal indicated that $70 \%$ of our participants were postmenopausal (not including younger women who had undergone surgically induced menopause). It is reasonable to assume that the prevalence of sexual distress and FSD symptoms should have been larger in our sample because they comprised more postmenopausal women who report greater frequencies of sexual problems (Dennerstein et al. 1994, 2003). Indeed, our frequencies are comparable to base rates for sexual problems in postmenopausal women (e.g. Dennerstein et al. 2003). The age-related effects on sexual function reported here and elsewhere indicate that caution be exercised in generalizing the current findings to other age groups. The effect of being single $(18 \%)$ was minimized by solely including females who reported some sexual activity during the time period included in our analyses (the past 4 weeks). We were unable to assess some potential confounders such as, for example, duration of relationship because of unavailability of the data. Duration of relationship might substantially impact relationship dissatisfaction and thus confound the association between sexual distress and relationship dissatisfaction. It should be noted that we used simple and restricted measures of relationship satisfaction, experiences of abuse, and impact of medical conditions and psychopathology instead of standardized items in most cases. Nevertheless, using these somewhat limited items led to results that were consistent with previous findings.

We cannot exclude the possibility that our data are affected by reporting biases given the sensitive nature of the questions, leading to some underestimation of sexual distress and FSD symptoms. However, although our response rates might seem low (50\%), they are in fact respectable relative to other epidemiological-level sex surveys (Bailey et al. 2000; Dunne et al. 2000; Hayes et al. 2008; Witting et al. 2008). Dunne et al. (2000) also reported that surveys of sexual behaviour may overestimate sexual liberalism, activity and dysfunction (in reporting) but that this bias does not seriously compromise population estimates, as judged by the pattern of effect sizes.

In summary, our results do not support the view that sexual distress is a necessary indicator of pathological sexual functioning in women at the aetiological or correlational level. Rather, unique environmental factors perhaps related to general psychological health and relationship satisfaction seem to influence both sexual distress and FSD. Further work is needed to confirm whether these associations are also the result of genetic or non-genetic confounding. Overall, our findings tentatively indicate that sexual distress might be less a consequence of FSD and more related to general anxiety and worry surrounding a woman's sexuality and relationship, independent from her actual level of sexual functioning. If confirmed in further research, mental health professionals may want to consider including generic therapeutic interventions for anxiety as part of a package of treatment for women presenting with FSD symptoms so as to reduce maladaptive thinking patterns related to sexual responses.

\section{Acknowledgements}

We acknowledge financial support from the Wellcome Trust, the Department of Health through the National Institute for Health Research (NIHR) comprehensive Biomedical Research Centre award to Guy's and St Thomas' National Health Service (NHS) Foundation Trust in partnership with King's College London, and the Chronic Disease Research Foundation.

\section{Declaration of Interest}

None.

\section{References}

Abdo CH, Oliveira WM, Moreira ED, Fittipaldi JA (2004).

Prevalence of sexual dysfunctions and correlated conditions in a sample of Brazilian women - results of the Brazilian study on sexual behavior (BSSB). International Journal of Impotence Research 16, 160-166.

Althof S (2001). My personal distress over the inclusion of personal distress. Journal of Sex and Marital Therapy 27, 123-125.

Andrews T, Hart DJ, Snieder H, de Lange M, Spector TD, MacGregor AJ (2001). Are twins and singletons comparable? A study of disease-related and lifestyle characteristics. Twin Research 4, 464-477.

APA (1994). Diagnostic and Statistical Manual of Mental Disorders, 4th edn. American Psychiatric Association: Washington, DC. 
Bailey JM, Dunne MP, Martin NG (2000). Genetic and environmental influences on sexual orientation and its correlates in an Australian twin sample. Journal of Personality and Social Psychology 78, 524-536.

Bancroft J, Loftus J, Long JS (2003). Distress about sex: a national survey of women in heterosexual relationships. Archives of Sexual Behavior 32, 193-208.

Basson R, Berman J, Burnett A, Derogatis L, Ferguson D, Fourcroy J, Goldstein I, Graziottin A, Heiman J, Laan E, Leiblum S, Padma-Nathan H, Rosen R, Segraves K, Segraves RT, Shabsigh R, Sipski M, Wagner G, Whipple B (2000). Report of the international consensus development conference on female sexual dysfunction: definitions and classifications. Journal of Urology 163, 888-893.

Basson R, Althof S, David S, Fugl-Meyer K, Goldstein I (2004). Summary of the recommendations on sexual dysfunctions in women. Journal of Sexual Medicine 1, 24-34.

Burri A, Cherkas L, Spector T (2009). Emotional intelligence and its association with orgasm frequency in women. Journal of Sexual Medicine 6, 1930-1937.

Burri A, Spector T (in press). Recent and lifelong sexual dysfunction in a female UK population sample: prevalence and risk factors. Journal of Sexual Medicine.

Dawood K, Kirk KM, Bailey JM, Andrews PW, Martin NG (2005). Genetic and environmental influences on the frequency of orgasm in women. Twin Research 8, 27-33.

Dennerstein L, Alexander JL, Kotz K (2003). The menopause and sexual functioning: a review of the population-based studies. Annual Review of Sex Research 14, 64-82.

Dennerstein L, Hayes RD (2005). Confronting the challenges: epidemiological study of female sexual dysfunction and the menopause. Journal of Sexual Medicine 2, 118-132.

Dennerstein L, Smith A, Morse C, Burger H (1994). Sexuality and the menopause. Journal of Psychosomatic Obstetrics and Gynecology 15, 59-66.

Derogatis L, Clayton A, Lewis-D'Agostino D, Wunderlich G, Fu Y (2008). Validation of the female sexual distress scale - revised for assessing distress in women with hypoactive sexual desire disorder. Journal of Sexual Medicine 5, 357-364.

Derogatis LR, Rosen R, Leiblum S, Burnett A, Heiman J (2002). The Female Sexual Distress Scale (FSDS): initial validation of a standardized scale for assessment of sexually related personal distress in women. Journal of Sex and Marital Therapy 28, 317-330.

Dunn KM, Croft PR, Hackett GI (1999). Association of sexual problems with social, psychological, and physical problems in men and women: a cross sectional population survey. Journal of Epidemiology and Community Health 53, 144-148.

Dunn KM, Cherkas LF, Spector TD (2005). Genetic influences on variation in female orgasmic function: a twin study. Biology Letters 22, 260-226.

Dunne MP, Bailey JM, Kirk KM, Martin NG (2000). The subtlety of sex atypicality. Archives of Sexual Behavior 29, 549-565.

Foa EB, Huppert JD, Kichic R, Hajcak G, Salkovskis PM (2002). The Obsessive-Compulsive Inventory: development and validation of a short version. Psychological Assessment 14, 485-496.

Frohlich P, Meston C (2002). Sexual functioning and self-reported depressive symptoms among college women. Journal of Sex Research 39, 321-325.

Graham CA (2010). The DSM diagnostic criteria for female sexual arousal disorder. Archives of Sexual behavior 39, 240-255.

Hayes RD, Dennerstein L, Bennett CM, Sidat M, Gurrin LC, Fairley CK (2008). Risk factors for female sexual dysfunction in the general population: exploring factors associated with low sexual function and sexual distress. Journal of Sexual Medicine 5, 1681-1693.

Hu LT, Bentler PM (1995). Evaluating model fit. In Structural Equation Modelling: Concepts, Issues and Applications (ed. R. H. Hoyle), pp. 76-99. Sage: Thousand Oaks, CA.

King M, Holt V, Nazareth I (2007). Women's views of their sexual difficulties: agreement and disagreement with clinical diagnoses. Archives of Sexual Behavior 36, 281-288.

Loehlin JC (1995). The Cholesky approach: a cautionary note. Behavior Genetics 26, 19-26.

Masters WH, Johnson VE (1966). Human Sexual Response. Little Brown: Boston.

Meston CM (2003). Validation of the Female Sexual Function Index (FSFI) in women with female orgasmic disorder and in women with hypoactive sexual desire disorder. Journal of Sex and Marital Therapy 29, 39-46.

Neale MC, Boker SM, Xie G, Maes HH (2006). Mx: Statistical Modeling, 7th edn. Virginia Commonwealth University: Richmond, VA.

Neale MC, Cardon LR (1992). Methodology for Genetic Studies of Twins and Families. Kluwer Academic: Dordrecht, The Netherlands.

Oberg K, Fugl-Meyer AR, Fugl-Meyer KS (2004). On categorization and quantification of women's sexual dysfunctions: an epidemiological approach. International Journal of Impotence Research 16, 261-269.

Peterson RA, Plehn K (1999). Measuring anxiety sensitivity. In Anxiety Sensitivity: Theory, Research, and Treatment of the Fear of Anxiety (ed. S. Taylor), pp. 61-81. Erlbaum: Mahwah, NJ, 1999.

Peterson RA, Reiss RJ (1992). Anxiety Sensitivity Index Manual (2nd edn). International Diagnostic Systems: Worthington, OH, 1992.

Petrides KV, Furnham A (2006). The role of trait emotional intelligence in a gender-specific model of organizational variables. Journal of Applied Social Psychology 36, 552-569.

Posthuma D, Beem AL, de Geus EJC, van Baal GCM, von Hjelmborg JB, Iachine I, Boomsma DI (2003). Theory and practice in quantitative genetics. Twin Research 6, 361-376.

Reiss S, Peterson RA, Gursky M, McNally R (1986). Anxiety, sensitivity, anxiety frequency, and the prediction of fearfulness. Behaviour Research and Therapy 24, 1-8.

Rosen R, Brown C, Heiman J, Leiblum S, Meston C, Shabsigh R, Ferguson D, D'Agostino R (2000). The Female Sexual Function Index (FSFI) : a multidimensional self-report instrument for the assessment of female sexual function. Journal of Sex and Marital Therapy 26, 191-208. 
Segraves R, Balon R, Clayton A (2007). Proposal for changes in diagnostic criteria for sexual dysfunctions. Journal of Sexual Medicine 3, 567-580.

Shifren JL, Monz BU, Russo PA, Segreti A, Johannes CB (2008). Sexual problems and distress in United States women. Obstetrics and Gynecology 112, 970-978.

Spector T, Williams F (2006). The UK Adult Twin Registry (TwinsUK). Twin Research and Human Genetics 9, 899-906.

StataCorp (2007). STATA Statistical Software: Release 10. Stata Corporation: College Station, TX.

Stephenson KR, Meston CM (2010). When are sexual difficulties distressing for women? The selective protective value of intimate relationships. Journal of Sexual Medicine 7, 3683-3694.

Wiegel M, Meston C, Rosen R (2005). The Female Sexual Function Index (FSFI) : cross-validation and development of clinical cut-off scores. Journal of Sex and Marital Therapy 31, 1-20.

Witting K, Santilla P, Rijsdijk F, Varjonen M, Stern P, Johansson A, von der Prahlen B, Alanko K, Sandnabba NK (2009). Correlated genetic and non-shared environmental influences account for the co-morbidity between female sexual dysfunctions. Psychological Medicine 26, 1-8.

Witting K, Santtila P, Varjonen M, Jern P, Johansson A, von der Pahlen B, Sandnabba K (2008). Female sexual dysfunction, sexual distress, and compatibility with partner. Journal of Sexual Medicine 5, 2587-2599.

Woods SA, Hampson SE (2005). Measuring the Big Five with single items using a bipolar response scale. European Journal of Personality 19, 373-390. 